\title{
Influência da sazonalidade na incidência de fissuras labiopalatinas em um centro de referência no Oeste do Paraná
}

\author{
Influence of seasonality on the incidence of cleft lip and palate in a reference center in western \\ Paraná
}

Influencia de la estacionalidad en la incidencia de labio y paladar hendido en un centro de referencia del oeste de Paraná

Recebido: 10/01/2022 | Revisado: 19/01/2022 | Aceito: 21/01/2022 | Publicado: 23/01/2022

\author{
Andressa Fernanda Luiz \\ ORCID: https://orcid.org/0000-0002-2735-7224 \\ Universidade Estadual do Oeste do Paraná, Brasil \\ E-mail: Andressa.luiz.974@gmail.com \\ Luciana Paula Grégio d'Arce Rodrigues \\ ORCID: https://orcid.org/0000-0002-8428-5319 \\ Universidade Estadual do Oeste do Paraná, Brasil \\ E-mail: lucianapgd@yahoo.com \\ Natani Ribeiro Demarco \\ ORCID: https://orcid.org/0000-0002-4882-1604 \\ Universidade Estadual do Oeste do Paraná, Brasil \\ E-mail: nnaat_@hotmail.com
}

\begin{abstract}
Resumo
Objetivo: O objetivo deste estudo foi verificar, com base na data de concepção, se existe uma influência da sazonalidade com o aumento do risco de desenvolvimento de fendas orofaciais, relacionando-o com o gênero. Metodologia: Estudo documental retrospectivo conduzido através da compilação de dados secundários de pacientes atendidos entre os anos de 2013 e 2019, em um centro de referência. Só foram incluídos pacientes com fissura labial e/ou palatina isolada. $\mathrm{O}$ teste $\mathrm{X}^{2}$ de aderência e o teste $\mathrm{X}^{2}$ de independência seguido, quando necessário, do pós-teste dos resíduos ajustados, foram utilizados para analisar a diferença estatística e a associação entre as variáveis. Os grupos foram ordenados considerando o gênero e a sazonalidade, divididos de acordo com as estações do ano: outono, inverno, primavera e verão. Resultados: 181 pacientes preencheram o critério de inclusão. A idade gestacional média foi de 38,4 semanas. A história familiar foi negativa em 114 (63\%) casos. Do total, 51 concepções $(28,18 \%)$ ocorreram na Primavera, $46(25,41 \%)$ no Inverno, $42(23,20 \%)$ no Outono, e $42(23,20 \%)$ no Verão. Não houve diferença significativa na incidência de fissuras orofaciais entre as 4 estações do ano, nem quando se relacionou a distribuição sazonal com o gênero. Conclusão: Não foi encontrada qualquer associação da sazonalidade com o desenvolvimento de fissuras orofaciais, considerando a data de concepção. Não foi encontrada diferença significativa ao relacionar a distribuição sazonal com o gênero.
\end{abstract}

Palavras-chave: Fenda labial; Fissura palatina; Estações do ano; Anormalidades craniofaciais.

\begin{abstract}
Purpose: The aim of this study was to verify, based on the date of conception, if there is an influence of seasonality with the increased risk of developing orofacial clefts, relating it to gender. Methodology: A retrospective documental study conducted by compiling secondary data of patients seen between the years 2013 and 2019, in a referral center. Only patients with isolated cleft lip and/or palate were included. The $\mathrm{X}^{2}$ test of adherence and the $\mathrm{X}^{2}$ test of independence followed, when necessary, by the post-test of adjusted residuals, were used to analyze the statistical difference and the association between the variables. The groups were ordered considering gender and seasonality, divided according to the seasons of the year: fall, winter, spring and summer. Results: 181 patients met the inclusion criteria. The mean gestational age was 38.4 weeks. Family history was negative in $114(63 \%)$ cases. Of the total, 51 conceptions $(28.18 \%)$ occurred in spring, $46(25.41 \%)$ in winter, $42(23.20 \%)$ in fall, and $42(23.20 \%)$ in summer. There was no significant difference in the incidence of orofacial clefts among the 4 seasons of the year, nor when the seasonal distribution was related to gender. Conclusion: No association was found between seasonality and the development of orofacial clefts, considering the date of conception. No significant difference was found when relating seasonal distribution to gender.
\end{abstract}

Keywords: Cleft lip; Cleft palate; Seasons; Craniofacial abnormalities. 


\begin{abstract}
Resumen
El objetivo de este estudio fue verificar, a partir de la fecha de concepción, si existe una influencia de la estacionalidad con el aumento del riesgo de desarrollar hendiduras orofaciales, relacionándolo con el género. Metodología: Estudio documental retrospectivo realizado mediante la recopilación de datos secundarios de pacientes atendidos entre los años 2013 y 2019, en un centro de referencia. Sólo se incluyeron pacientes con labio y/o paladar hendido aislados. Para analizar la diferencia estadística y la asociación entre las variables se utilizó la prueba $\mathrm{X}^{2}$ de adherencia y la prueba $\mathrm{X}^{2}$ de independencia, seguidas, cuando fue necesario, de la prueba posterior de residuos ajustados. Los grupos se ordenaron teniendo en cuenta el género y la estacionalidad, divididos según las estaciones del año: otoño, invierno, primavera y verano. Resultados: 181 pacientes cumplieron los criterios de inclusión. La edad gestacional media fue de 38,4 semanas. Los antecedentes familiares fueron negativos en 114 (63\%) casos. Del total, 51 concepciones $(28,18 \%)$ se produjeron en primavera, $46(25,41 \%)$ en invierno, $42(23,20 \%)$ en otoño y $42(23,20 \%)$ en verano. No hubo diferencias significativas en la incidencia de hendiduras orofaciales entre las 4 estaciones del año, ni cuando la distribución estacional se relacionó con el género. Conclusión: No se encontró ninguna asociación entre la estacionalidad y el desarrollo de hendiduras orofaciales, teniendo en cuenta la fecha de concepción. No se encontraron diferencias significativas al relacionar la distribución estacional con el género.
\end{abstract}

Palabras clave: Labio leporino; Fisura del paladar; Estaciones del año; Anomalías craneofaciales.

\title{
1. Introdução
}

As fendas orofaciais são decorrentes de falhas no desenvolvimento ou na maturação dos processos faciais durante a vida embrionária, e estão entre os defeitos estruturais congênitos mais frequentes, afetando cerca de 1,43:/1.000 recémnascidos no mundo todo (Costa, Takeshita e Farah, 2013; Dixon et al., 2011) A prevalência de fissura labial e / ou palatina é conhecida por variar entre as raças e regiões geográficas (Cooper, Ratay e Marazita, 2006). No Brasil, nasce um bebê fissurado a cada 650 nascimentos (Governo do Brasil, 2017).

A etiologia das fendas orofaciais ainda é em grande parte desconhecida, no entanto, sabe-se que fatores genéticos e ambientais, além forte interferência de fatores étnicos, são contribuintes importantes para o desenvolvimento dessa anomalia (Borges et al., 2014, Schoenwolf et al., 2016).

Os fatores exógenos/ambientais que podem estar associados a incidência da fissura são diversos, e a variação sazonal é um deles. A variação sazonal pode ser definida como diferenças devidas a influências periódicas, temporais e externas, nomeadamente a determinada época ou estação do ano (Dodge, 2006). O estudo das tendencias sazonais de anomalias congênitas levam em consideração diversos fatores, como intensidade da luz UV, temperatura, radiação, exposição a químicos agrícolas, ciclo de doenças infecciosas, disposição de alimentos, nutrição materna, entre outros (Krost \& Schubert, 2006). A prevalência da ocorrência de fissuras orofaciais em certa estação do ano (primavera, verão, outono e inverno) pode ajudar no entendimento do papel do ambiente sobre esse defeito congênito. Apesar disso, os resultados variam amplamente, possivelmente devido as diferenças geográficas, climáticas e socioeconômicas.

O objetivo deste estudo foi verificar, com base na data de concepção, se existe uma influência da sazonalidade com o aumento do risco de desenvolvimento de fendas orofaciais, relacionando-o com o gênero, no Centro de Atenção e Pesquisa em Anomalias Craniofaciais (CEAPAC), que é um centro de referência para a região oeste do Paraná.

\section{Metodologia}

Este estudo foi revisto e aprovado pelo Comitê de Ética (Parecer 3.441.096, CAEE: 15663919.0.0000.0107). Para a coleta de dados, foram seguidos os princípios delineados na Declaração de Helsinque.

Este é um estudo documental retrospectivo conduzido através da compilação de dados secundários de pacientes atendidos no CEAPAC, localizado na cidade de Cascavel-PR, entre os anos de 2013 e 2019.

Os dados recolhidos incluem: data de nascimento, idade, sexo, origem, duração da gestação em semanas, história familiar, tipo de fenda, e outras malformações presentes. Para determinar a data estimada de concepção, esta foi calculada 
reduzindo a data de nascimento pela idade gestacional e adicionando 2 semanas. Para a composição da amostra, só foram incluídos pacientes com fissura labial e/ou palatina, ou seja, sem apresentar qualquer outra malformação congênita e/ou sem associação com qualquer síndrome genética. O tipo de fenda foi classificado de acordo com o modelo proposto por Spina et al. (1972), utilizando o forame incisivo como referência anatômica.

Foram realizadas análises estatísticas utilizando o programa licenciado XLSTat Versão 2017 (Addinsoft). O teste X² de aderência e o teste $\mathrm{X}^{2}$ de independência seguido, quando necessário, do pós-teste dos resíduos ajustados, foram utilizados para analisar a diferença estatística e a associação entre as variáveis. Um valor $\mathrm{P}$ de $\leq 0,05$ foi utilizado como significância estatística. Os grupos foram ordenados considerando o gênero e a sazonalidade, divididos de acordo com as estações do ano: Outono (20 de Março a 21 de Junho), Inverno (21 de Junho a 23 de Setembro), Primavera (23 de Setembro a 22 de Dezembro), e Verão (22 de Dezembro a 20 de Março).

\section{Resultados}

Ao todo, 181 pacientes preencheram o critério de inclusão, também foram excluídos deste estudo os casos em que a idade gestacional era desconhecida, o que pode explicar o baixo tamanho da amostra. A idade gestacional média foi de 38,4 semanas. A história familiar foi negativa em 114 (63\%) casos. As percentagens de todas as concepções foram calculadas, 51 $(28,18 \%)$ ocorreram na Primavera, $46(25,41 \%)$ no Inverno, $42(23,20 \%)$ no Outono, e $42(23,20 \%)$ no Verão. Os testes estatísticos não mostraram uma associação significativa de variação sazonal com o aparecimento de fissuras, considerando a data de concepção dos indivíduos estudados (Tabela 1). Também não foi encontrada qualquer diferença significativa quando se relacionou a distribuição sazonal com o gênero. (Tabela 2).

Tabela 1. Frequências absolutas (FA) e relativas (FR\%) de incidência de fissuras por estação, tendo em conta a data de concepção. P-valor do teste de aderência $\mathrm{X}^{2}$.

\begin{tabular}{|c|c|c|c|c|}
\hline Variável & Categoria & FA & FR \% & p-valor \\
\hline \multirow{4}{*}{ Estação do ano } & Primavera & 51 & 28,18 & \multirow{4}{*}{0,656} \\
\hline & Inverno & 46 & 25,41 & \\
\hline & Outono & 42 & 23,20 & \\
\hline & Verão & 42 & 23,20 & \\
\hline
\end{tabular}

Fonte: Autores (2021). 
Tabela 2. Frequências absolutas (FA) e frequências relativas (FR\%) da distribuição sazonal por gênero. P-valor do teste de independência $\mathrm{X}^{2}$.

\begin{tabular}{|ccccccc|}
\hline & & \multicolumn{2}{c}{ Feminino } & \multicolumn{2}{c|}{ Masculino } \\
\hline Variável & Categoria & FA & FR \% & FA & FR \% & p-valor \\
\hline \multirow{2}{*}{$\begin{array}{c}\text { Estação do } \\
\text { Ano }\end{array}$} & Inverno & 12 & 19,05 & 34 & 28,81 & \\
\cline { 2 - 6 } & Outono & 14 & 22,22 & 28 & 23,73 & \multirow{2}{*}{0,368} \\
& Primavera & 22 & 34,92 & 29 & 24,58 & \\
& Verão & 15 & 23,81 & 27 & 22,88 & \\
\hline
\end{tabular}

Fonte: Autores (2021).

\section{Discussão}

Neste estudo, procuramos descobrir se existe uma influência da sazonalidade e o aumento do risco de desenvolvimento de fissuras orofaciais. Os testes estatísticos não mostraram uma associação significativa de variação sazonal com o aparecimento de fissuras, considerando a data de concepção dos indivíduos estudados (p=0,656) (Tabela I). Um estudo realizado no estado de Minas Gerais com 361 pacientes e um grupo controle $(n=481)$, não encontrou diferenças significativas na variação sazonal considerando o mês de nascimento. Entretanto, o grupo controle houve mais partos nos meses de fevereiro e agosto, e no grupo fissurado, nos meses de julho e agosto (Aquino et al., 2017).

Krost e Schubert (2006), em uma pesquisa na Alemanha, com base na data de concepção, provaram um máximo significativo e um risco relativo de 2,6 vezes de pessoas do sexo feminino que conceberam durante a primavera, verão e outono de desenvolver fissura labial. Em Montreal, foi possível observar diferenças significativas, sendo o sexo masculino com maior tendência de nascimentos no verão (Fraser \& Gwyn, 1998). Um estudo realizado na Holanda, também considerando a data de concepção, mostrou uma variação sazonal significativa em relação ao sexo masculino com mais concepções na primavera e principalmente em maio (Van der Lek et al., 2021). Neste estudo, não foi observada nenhuma diferença significativa de sexo com período de concepção sazonal.

O consumo alimentar materno e o estado nutricional desempenham um importante fator no desenvolvimento de fissuras orofaciais não sindrômicas (Krapels et al., 2006, Silva et al., 2018). Devido ao aumento da demanda de nutrientes para o crescimento fetal e tecidos maternos, as mulheres grávidas são propensas a desenvolver uma deficiência de folato, uma vitamina do complexo B encontrada em vegetais de folha, feijão, vísceras, leite, e em algumas frutas como abacates e laranjas (Maia et al., 2020). No Brasil, a Agência Nacional de Vigilância Sanitária (ANVISA) estabeleceu uma dose diária de 0,6 mg/dia para mulheres grávidas de ingestão de ácido fólico, além de incluir a fortificação obrigatória de ácido fólico em cereais fabricados, tais como farinha, arroz, pães, macarrão e produtos derivados do milho comercializados no Brasil (Santos \& Pereira, 2007). O estudo realizado por Souza e Raskin (2013) e Webhy e colaboradores (2013), observou resultados satisfatórios relativos à diminuição de casos de fendas não sindrómicas no Brasil após a resolução do Ministério da Saúde em 2004, que tornou obrigatória a fortificação da farinha de trigo com 1,5 mg/kg de ácido fólico. Altoé et al. (2020) constataram um aumento de 2,94 a 3,17 na chance de ocorrência de fissura oral em mães que não tiveram a devida suplementação de ácido fólico no primeiro trimestre de gestação. É importante salientar que os hábitos alimentares brasileiros mais tradicionais incluem o consumo anual de arroz, feijão e carne, bem como a presença constante de derivados da farinha de trigo, o que nos 
permite inferir que o consumo de folato permanece constante durante todos os meses, independentemente da estação do ano.

Grande parte dos estudos acabam adotando a data de nascimento como base para os cálculos, no entanto, as malformações não cromossômicas surgem no primeiro trimestre de gestação, o que torna essas pesquisas menos informativas e conflitantes, como os trabalhos de Elliot e Beveridge (2008), Sakran e colaboradores (2021), Hao et al. (2021), dentre outros. A amostra deste presente trabalho mostrou-se pequena devido ao critério de dados de gestação, justamente porque tentou-se calcular exatamente a data de concepção, para determinar o primeiro trimestre gestacional, período no qual são formadas as fissuras.

O estudo apresentou certas limitações quanto a obtenção de dados. Cabe destacar a dificuldade da coleta de dados nas fichas de atendimento, já que no decorrer dos anos ocorreram mudanças no modelo de distribuição das informações, além da falta de dados em alguns casos. Também deve-se considerar a confiabilidade dos dados obtidos. Outros fatores ambientais que poderiam influenciar a pesquisa não foram registrados. Cabe ressaltar a escassez de literatura referente as fissuras orofaciais, especialmente relacionado a sua etiologia em estudos no Brasil.

\section{Conclusão}

Neste estudo, não foi encontrada qualquer associação da sazonalidade com o desenvolvimento de fissuras orofaciais, considerando a data de concepção. Não foi encontrada diferença significativa ao relacionar a distribuição sazonal com o gênero. Os resultados deste estudo mostram a necessidade de investigações na população brasileira, especialmente sobre o envolvimento de fatores ambientais no desenvolvimento de malformações congênitas, especialmente fissuras orofaciais.

\section{Referências}

Altoé, R. S. et al (2020). Influence of parental exposure to risk factors in the occurrence of oral clefts. J. Dent., v.21, n.2, p.119- 126

Borges, A. R.; Mariano, L.; Sá, J.; Medrado, A. P.; Veiga, P. C.; Reis, S. R. A. (2014). Fissuras labiais e/ou palatinas não sindrômicas: determinantes ambientais e genéticos. Revista Bahiana de Odontologia. 5(1):48-58.

Cooper, M. E., Ratay, J. S., Marazita, M. L. (2006). Asian oral-facial cleft birth prevalence. Cleft Palate Craniofac J. 43(5):580-9.

Costa, R. R.; Takeshita, W. M.; Farah, G. J (2013). Levantamento epidemiológico de fissuras labiopalatais no município de Maringá e região. Rev. Assoc. Paul. Cir. Dent., 67(1): 40-44.

Aquino, S. N.; Machado, R. A.; Paranaíba, L. M. R.; Martelli, D. R.; Popoff, D. A. V.; Swerts, M. S. O. et al. (2017). A review of seasonality of cleft births The Brazil experience. J Oral Biol Craniofac Res.7(1):2-6.

Dixon, M. J.; Marazita, M.; Beaty, T. H.; Murray, J. C. (2011). Cleft lip and palate: understanding genetic and environmental influences. Nat Rev Genet., 12(3):167-78.

Dodge, Y. (2006). The Oxford Dictionary of Statistical Terms. Oxford: Oxford University Press.

Elliott, R. F.; Jovic, G.; Beveridge, M. (2008). Seasonal variation and regional distribution of cleft lip and palate in Zambia. Cleft Palate Craniofac J. 45(5):533-8.

Fraser, F. C. \& Gwyn, A. (1998). Seasonal variation in birth date of children with cleft lip. Teratology. 57(2):93-5.

Governo do Brasil, 2017. Um em cada 650 bebês nasce com fissura labiopalatal no país. http://www.brasil.gov.br/noticias/saude/2016/11/um-em-cada-650bebes-nasce-com-fissuralabiopalatal- no-pais.

Hao, Y.; Zhuang, D.; Jiao, X. (2021). Seasonal Variation of Nonsyndromic Orofacial Clefts in Northern Chinese Population, Journal of Craniofacial Surgery.

Krapels, I. P.; Vermeij-Keers, C.; Müller, M.; de Klein, A.; Steegers-Theunissen, R. P. (2006). Nutrition and genes in the development of orofacial clefting. Nutr Rev.64(6):280-8

Krost, B. \& Schubert, J. (2006). Influence of season on prevalence of cleft lip and palate. Int J Oral Maxillofac Surg. 35(3):215-8.

Maia, C. S.; Junior, J. R. A. Q.; Medeiros, J. P.; Tenório, F. C. A. M. et al. (2020). Metabolismo do ácido fólico e suas ações na embriogênese. Braz. J. of Develop.,Curitiba, v.6, n.8,p. 57002-57009

Sakran, K. A.; Mashrah, M. A.; Al-Rokhami, R. K.; Hsieh, T.; Huang, H.; Alkebsi, K.; et al. (2021) Nonsyndromic Oral clefts and associated risk factors in Gansu Province, Northwestof China. J Oral Maxillofac Surg Med Pathol. 33(5): 494-499 
Research, Society and Development, v. 11, n. 2, e20111225577, 2022

(CC BY 4.0) | ISSN 2525-3409 | DOI: http://dx.doi.org/10.33448/rsd-v11i2.25577

Santos, L. M. P. \& Pereira, M. Z. (2007). Efeito da fortificação com ácido fólico na redução dos defeitos do tubo neural. Cadernos de Saúde Pública. 23(1):17-24.

Schoenwolf, G. C. et al. (2016). Larsen embriologia humana. 5. ed. - Rio de Janeiro : Elsevier, 178 - 698

Silva, C. M., Pereira, M. C., Queiroz, T. B., \& Neves, L. T. (2018). O papel do ácido fólico na prevenção das fissuras labiopalatinas não sindrômicas: uma revisão integrative. Braz. Ap. Sci. Rev., Curitiba, v. 3, n. 1, p. 641-658.

Souza, J. \& Raskin, S. (2013). Clinical and epidemiological study of orofacial clefts. J. Pediatr. 89(2):137-144.

Spina, V.; Psillakis, J. M.; Lapa, F.S. (1972). Classificação das fissuras lábio-palatinas: sugestão de modificação. Rev. Hosp Clin Fac Med São Paulo. 27:5-6.

Van der Lek, L .M.; Pool, S. M. W.; de Jong, K.; Vermeij-Keers, C.; Mouës-Vink, C. M. (2021). Seasonal Influence on the Numbers of Gender-Related Orofacial Cleft Conceptions in the Netherlands. Cleft Palate Craniofac J.

Wehby, G. L.; Félix, T. M.; Goco, N.; Richieri-Costa, A.; Chakraborty, H.; Souza, J.; et al. (2013). High dosage folic acid supplementation, oral cleft recurrence, and fetal growth. Int J Environ Res Public Health. 10(2):590-605. 